(C) by Oldenbourg Wissenschaftsverlag, München

\title{
Crystal structure of bis(1,10-phenanthroline)copper(I) dichlorocuprate(I), $\left[\mathrm{Cu}\left(\mathrm{C}_{12} \mathrm{H}_{8} \mathrm{~N}_{2}\right)_{2}\right]\left[\mathrm{CuCl}_{2}\right]$
}

\author{
J.-G. Wang*, H.-X. Kang and X.-Y. Zheng \\ Luoyang Normal University, Department of Chemistry, Luoyang, Henan, 471022 P. R. China
}

Received October 3, 2005, accepted and available on-line December 3, 2005; CCDC no. 1267/1655

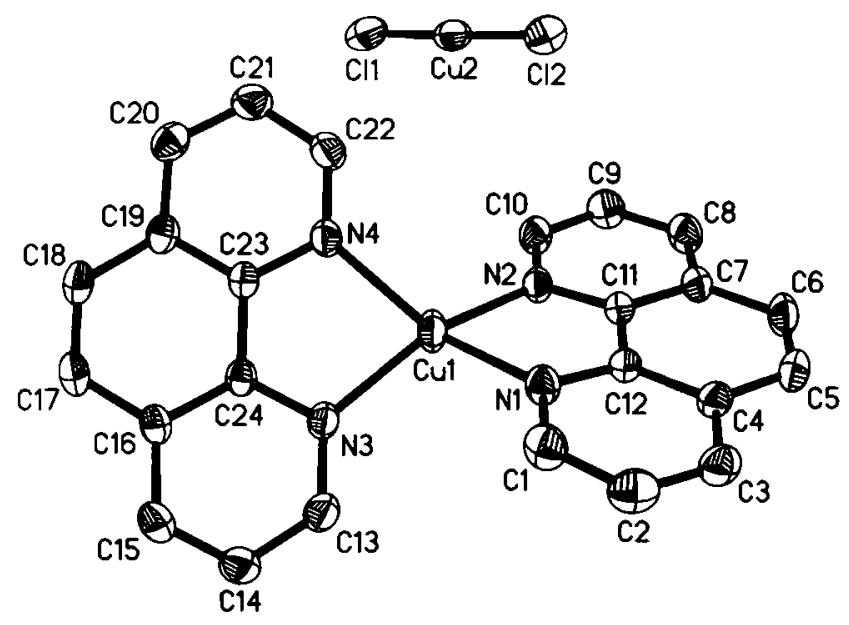

Abstract

$\mathrm{C}_{24} \mathrm{H}_{16} \mathrm{Cl}_{2} \mathrm{Cu}_{2} \mathrm{~N}_{4}$, monoclinic, $C 12 / c 1$ (no. 15), $a=19.709(3) \AA, b=14.830(3) \AA, c=15.208(3) \AA$, $\beta=103.096(2)^{\circ}, V=4329.5 \AA^{3}, Z=8, R_{\mathrm{gt}}(F)=0.027$, $w R_{\text {ref }}\left(F^{2}\right)=0.089, T=273 \mathrm{~K}$.

\section{Source of material}

The hydrothermal reaction of copper(II) dichloride (0.5 mmol) 1,10 -phenanthroline $(0.5 \mathrm{mmol})$ and $N$-benzenesulfonyl-L-glutamic acid ( $1 \mathrm{mmol})$ in an aqueous solution $(12 \mathrm{~mL})$ was carried out in a Teflon-lined stainless steel reactor. The mixture was heated at $165^{\circ} \mathrm{C}$ for three days, and then slowly cooled down to room temperature. A dark green solution was formed. Dark green prismatic crystals of the title complex were grown from the solution by slow evaporation for about six days and isolated (yield $48 \%$ ).

\section{Discussion}

Crystal structures of bis-chelated transition-metal 1,10-phenanthroline (phen) complexes containing the $\mathrm{Cu}(\mathrm{phen}){ }_{2}{ }^{+}$fragment have been extensively studied during last two decades [1-11]. However, complexes containing $\mathrm{Cu}(\text { phen })_{2}{ }^{+}$and $\mathrm{Cu}\left(\mathrm{Cl}_{2}\right)^{-}$subunits simultaneously have not been reported. During our investigation of the self-assemblies of metal ion and mixed ligands (1,10-phenanthroline and $N$-benzenesulfonyl-L-glutamic acid), we did not obtain the expected compound, but, instead, dark green prismatic crystals of bis(1,10-phenanthroline)copper(I) dichloro-cuprate(I), grew from the above solution on slow evaporation. The new complex has been characterized by single-crystal diffraction analysis. The crystal structure of the title compound is composed of discrete $\left[\mathrm{Cu}(\text { phen })_{2}\right]^{+}$cations and $\left[\mathrm{CuCl}_{2}\right]^{-}$anions. The coordination environments of the two copper ions are different. The central $\mathrm{Cu} 1$ ion is four-coordinated by the nitrogen do-

\footnotetext{
* Correspondence author (e-mail: wang_jiange@126.com)
}

nors from two bidentate phen ligands, forming two fivemembered chelated rings. The two rings are not in a good planarity with a dihedral angle of $48.1^{\circ}$. The four $\mathrm{Cul}-\mathrm{N}$ bond lengths are in the range of $2.057(2) \AA-2.092(2) \AA$ with an average value of $2.0745 \AA$, which are significantly longer than the averaged $\mathrm{Cu}(\mathrm{I})-\mathrm{N}$ distances of $\mathrm{Cu}$ (phen $)_{2}{ }^{+}$subunits in other compounds: [Cu(phen) $\left.)_{2}\right]\left[\mathrm{ClO}_{4}\right](2.049 \AA)[2],[\mathrm{Cu}($ phen) 2$] \mathrm{I}(2.044 \AA)$ [6], [Cu(phen) $\left.\left.)_{2}\right] \mathrm{CuBr}_{2}\right](2.039 \AA)$ [2]. The central $\mathrm{Cu} 2$ ion is twocoordinated by two chlorine atoms with $\mathrm{Cu} 2-\mathrm{Cl}$ bond lengths are $2.0882(9) \AA$ and $2.0958(9) \AA$, respectively, the angle of $\mathrm{Cl}-\mathrm{Cu}-\mathrm{Cl}$ is $178.75(3)^{\circ}$, which are close to those in the complex $\left[\mathrm{Cu}(\mathrm{II})(\text { phen })_{2} \mathrm{Cl}\right]\left[\mathrm{Cu}(\mathrm{I}) \mathrm{Cl}_{2}\right]$ [9]. In the arrangement of the unit cell, there exist orderly aromatic ring stacking interactions between phen ligands among the molecules of the complex. Six phen atoms $(\mathrm{C1}, \mathrm{C} 2, \mathrm{C} 3, \mathrm{C} 4, \mathrm{C1} 2, \mathrm{~N} 1)$ form weak $\pi-\pi$ stacking with other phen atoms $\left(\mathrm{C7}^{\prime}, \mathrm{Cl}^{\prime}, \mathrm{C}^{\prime}, \mathrm{ClO}^{\prime}, \mathrm{Cl}^{\prime}, \mathrm{N}^{\prime}\right)$ in a neighboring molecule with centroid distance of $3.785 \AA$ and dihedral angle of $3.7^{\circ}$. Such stacking expands along the $c$ axis to form a one-dimensional chain structure. The $\boldsymbol{N}$-benzenesulfonyl-L-glutamic acid obviously acts as reducing agent in the reduction of $\mathrm{Cu}(\mathrm{II}) / \mathrm{Cu}(\mathrm{I})$. The mechanistic details of the redox process are not completely clear. A similar phenomenon has been observed for the formation of $\mathrm{Cu}(\mathrm{II})(\text { phen })_{2} \mathrm{Cl} \cdot \mathrm{Cu}\left(\mathrm{ICl}_{2}[10]\right.$.

Table 1. Data collection and handling.

$\begin{array}{ll}\text { Crystal: } & \begin{array}{l}\text { dark green prism, } \\ \text { size } 0.18 \times 0.34 \times 0.35 \mathrm{~mm}\end{array} \\ \text { Wavelength: } & \text { Mo } K_{a} \text { radiation }(0.71073 \AA) \\ \mu: & 22.32 \mathrm{~cm}^{-1} \\ \text { Diffractometer, scan mode: } & \text { Bruker SMART CCD, } \varphi / \omega \\ 2 \theta_{\text {max }}: & 55^{\circ} \\ N(h k l)_{\text {measured, }} N(h k l)_{\text {unique }}: & 14321,4943 \\ \text { Criterion for } I_{\text {obs }}, N(h k l)_{\text {gt }}: & I_{\text {obs }}>2 \sigma\left(I_{\text {obs }}\right), 3875 \\ N(\text { param })_{\text {refined: }} & 353 \\ \text { Programs: } & \text { SHELXS-97 [12], SHELXL-97 [13] }\end{array}$

Table 2. Atomic coordinates and displacement parameters (in $\AA^{2}$ ).

\begin{tabular}{|c|c|c|c|c|c|}
\hline Atom & Site & $x$ & $y$ & $z$ & $U_{\text {iso }}$ \\
\hline$H(1)$ & $8 f$ & $0.353(1)$ & $0.344(2)$ & $0.002(2)$ & $0.067(6)$ \\
\hline$H(2)$ & $8 f$ & $0.284(1)$ & $0.472(2)$ & $-0.034(2)$ & $0.090(8)$ \\
\hline H(3) & $8 f$ & $0.330(1)$ & $0.610(2)$ & $0.010(2)$ & $0.063(6)$ \\
\hline H(5) & $8 f$ & $0.437(1)$ & $0.701(1)$ & $0.087(2)$ & $0.061(6)$ \\
\hline H(6) & $8 f$ & $0.553(2)$ & $0.704(2)$ & $0.165(2)$ & $0.077(8)$ \\
\hline H(8) & $8 f$ & $0.659(1)$ & $0.616(2)$ & 0.249 (2) & $0.082(8)$ \\
\hline H(9) & $8 f$ & $0.712(1)$ & $0.480(2)$ & $0.285(2)$ & $0.080(7)$ \\
\hline$H(10)$ & $8 f$ & $0.650(1)$ & $0.350(2)$ & $0.237(1)$ & $0.055(6)$ \\
\hline H(13) & $8 f$ & $0.355(1)$ & $0.243(2)$ & $0.158(1)$ & $0.056(6)$ \\
\hline H(14) & $8 f$ & $0.296(1)$ & $0.116(2)$ & $0.172(2)$ & $0.068(7)$ \\
\hline
\end{tabular}


Table 2. Continued

\begin{tabular}{llllll}
\hline Atom & Site & $x$ & $y$ & $z$ & $U_{\text {iso }}$ \\
\hline H(15) & $8 f$ & $0.347(1)$ & $-0.023(2)$ & $0.166(2)$ & $0.069(7)$ \\
H(17) & $8 f$ & $0.449(1)$ & $-0.110(2)$ & $0.134(2)$ & $0.064(6)$ \\
H(18) & $8 f$ & $0.561(1)$ & $-0.105(2)$ & $0.113(2)$ & $0.063(6)$ \\
\hline
\end{tabular}

Table 2. Continued

\begin{tabular}{llllll}
\hline Atom & Site & $x$ & $y$ & $z$ & $U_{\text {iso }}$ \\
\hline$H(20)$ & $8 f$ & $0.663(1)$ & $-0.019(2)$ & $0.083(1)$ & $0.063(6)$ \\
$H(21)$ & $8 f$ & $0.714(1)$ & $0.120(2)$ & $0.073(2)$ & $0.078(7)$ \\
$H(22)$ & $8 f$ & $0.649(1)$ & $0.249(2)$ & $0.078(1)$ & $0.056(6)$
\end{tabular}

Table 3. Atomic coordinates and displacement parameters (in $\AA^{2}$ ).

\begin{tabular}{|c|c|c|c|c|c|c|c|c|c|c|}
\hline Atom & Site & $x$ & $y$ & $z$ & $U_{11}$ & $U_{22}$ & $U_{33}$ & $U_{12}$ & $U_{13}$ & $U_{23}$ \\
\hline $\mathrm{Cu}(1)$ & $8 f$ & $0.50167(1)$ & $0.29671(2)$ & $0.12114(2)$ & $0.0575(2)$ & $0.0293(1)$ & $0.0803(2)$ & $-0.00075(9)$ & $0.0111(1)$ & $-0.0008(1)$ \\
\hline $\mathrm{Cu}(2)$ & $8 f$ & $0.79877(1)$ & $0.29842(2)$ & $0.25853(2)$ & $0.0502(2)$ & $0.0433(2)$ & $0.0942(2)$ & $0.0005(1)$ & $0.0137(1)$ & $0.0072(1)$ \\
\hline$C(1)$ & $8 f$ & $0.79085(4)$ & $0.30201(4)$ & $0.39311(5)$ & $0.0757(4)$ & $0.0589(4)$ & $0.0914(4)$ & $-0.0088(3)$ & $0.0122(3)$ & $0.0149(3)$ \\
\hline $\mathrm{Cl}(2)$ & $8 f$ & $0.80467(4)$ & $0.29321(4)$ & $0.12275(5)$ & $0.0797(4)$ & $0.0638(4)$ & $0.0994(5)$ & $-0.0123(3)$ & $0.0294(3)$ & $-0.0047(3)$ \\
\hline$N(1)$ & $8 f$ & $0.43502(8)$ & $0.4020(1)$ & $0.0667(1)$ & $0.0477(8)$ & $0.0417(8)$ & $0.0523(8)$ & $-0.0031(6)$ & $0.0044(6)$ & $-0.0023(7)$ \\
\hline$N(2)$ & $8 f$ & $0.56378(7)$ & $0.40442(9)$ & $0.1740(1)$ & $0.0445(7)$ & $0.0334(7)$ & $0.0528(8)$ & $0.0007(6)$ & $0.0078(6)$ & $0.0032(6)$ \\
\hline$N(3)$ & $8 f$ & $0.44047(8)$ & $0.18961(9)$ & $0.1409(1)$ & $0.0458(8)$ & $0.0352(8)$ & $0.0563(9)$ & $0.0006(6)$ & $0.0089(6)$ & $-0.0055(6)$ \\
\hline $\mathbf{N}(4)$ & $8 f$ & $0.56660(8)$ & $0.19073(9)$ & $0.1013(1)$ & $0.0474(8)$ & $0.0347(8)$ & $0.0600(9)$ & $0.0008(6)$ & $0.0107(7)$ & $0.0036(6)$ \\
\hline$C(1)$ & $8 f$ & $0.3691(1)$ & $0.4010(2)$ & $0.0194(2)$ & $0.055(1)$ & $0.059(1)$ & $0.063(1)$ & $-0.011(1)$ & $0.0012(9)$ & $-0.010(1)$ \\
\hline$C(2)$ & $8 f$ & $0.3290(1)$ & $0.4784(2)$ & $-0.0024(2)$ & $0.043(1)$ & $0.084(2)$ & $0.060(1)$ & $0.003(1)$ & $-0.0018(9)$ & $0.002(1)$ \\
\hline$C(3)$ & $8 f$ & $0.3575(1)$ & $0.5606(2)$ & $0.0222(1)$ & $0.049(1)$ & $0.062(1)$ & $0.058(1)$ & $0.012(1)$ & $0.0087(8)$ & $0.011(1)$ \\
\hline$C(4)$ & $8 f$ & $0.42681(9)$ & $0.5658(1)$ & $0.0720(1)$ & $0.0455(9)$ & $0.046(1)$ & $0.049(1)$ & $0.0076(8)$ & $0.0129(7)$ & $0.0068(8)$ \\
\hline$C(5)$ & $8 f$ & $0.4615(1)$ & $0.6487(1)$ & $0.1009(2)$ & $0.062(1)$ & $0.036(1)$ & $0.077(1)$ & $0.0109(9)$ & $0.016(1)$ & $0.0067(9)$ \\
\hline$C(6)$ & $8 f$ & $0.5281(1)$ & $0.6498(1)$ & $0.1498(2)$ & $0.063(1)$ & $0.032(1)$ & $0.085(1)$ & $-0.0030(9)$ & $0.014(1)$ & $-0.0008(9)$ \\
\hline$C(7)$ & $8 f$ & $0.56560(9)$ & $0.5678(1)$ & $0.1758(1)$ & $0.0484(9)$ & $0.0364(9)$ & $0.056(1)$ & $-0.0044(7)$ & $0.0123(8)$ & $-0.0014(8)$ \\
\hline$C(8)$ & $8 f$ & $0.6343(1)$ & $0.5649(2)$ & $0.2284(2)$ & $0.053(1)$ & $0.047(1)$ & $0.071(1)$ & $-0.0118(9)$ & $0.0056(9)$ & $-0.007(1)$ \\
\hline$C(9)$ & $8 f$ & $0.6660(1)$ & $0.4838(2)$ & $0.2501(1)$ & $0.044(1)$ & $0.061(1)$ & $0.064(1)$ & $-0.0027(9)$ & $-0.0011(9)$ & $0.002(1)$ \\
\hline$C(10)$ & $8 f$ & $0.6292(1)$ & $0.4058(1)$ & $0.2224(1)$ & $0.048(1)$ & $0.045(1)$ & $0.062(1)$ & $0.0066(8)$ & $0.0040(8)$ & $0.0108(9)$ \\
\hline$C(11)$ & $8 f$ & $0.53285(8)$ & $0.4852(1)$ & $0.1494(1)$ & $0.0416(8)$ & $0.0335(8)$ & $0.0429(8)$ & $0.0004(7)$ & $0.0108(7)$ & $0.0028(6)$ \\
\hline$C(12)$ & $8 f$ & $0.46246(8)$ & $0.4839(1)$ & $0.0941(1)$ & $0.0418(8)$ & $0.0362(9)$ & $0.0419(9)$ & $0.0007(7)$ & $0.0108(7)$ & $0.0006(7)$ \\
\hline$C(13)$ & $8 f$ & $0.3758(1)$ & $0.1894(1)$ & $0.1536(2)$ & $0.050(1)$ & $0.046(1)$ & $0.069(1)$ & $0.0016(9)$ & $0.0128(9)$ & $-0.0121(9)$ \\
\hline$C(14)$ & $8 f$ & $0.3391(1)$ & $0.1111(2)$ & $0.1615(2)$ & $0.045(1)$ & $0.062(1)$ & $0.067(1)$ & $-0.0056(9)$ & $0.0139(9)$ & $-0.008(1)$ \\
\hline$C(15)$ & $8 f$ & $0.3693(1)$ & $0.0298(2)$ & $0.1559(1)$ & $0.051(1)$ & $0.051(1)$ & $0.062(1)$ & $-0.0133(9)$ & $0.0076(9)$ & $-0.0022(9)$ \\
\hline$C(16)$ & $8 f$ & $0.43715(9)$ & $0.0261(1)$ & $0.1399(1)$ & $0.0490(9)$ & $0.0374(9)$ & $0.0449(9)$ & $-0.0056(7)$ & $0.0018(7)$ & $-0.0020(7)$ \\
\hline$C(17)$ & $8 f$ & $0.4729(1)$ & $-0.0560(1)$ & $0.1315(1)$ & $0.061(1)$ & $0.034(1)$ & $0.062(1)$ & $-0.0058(9)$ & $0.0030(9)$ & $0.0011(8)$ \\
\hline$C(18)$ & $8 f$ & $0.5380(1)$ & $-0.0556(1)$ & $0.1162(1)$ & $0.060(1)$ & $0.0322(9)$ & $0.062(1)$ & $0.0064(8)$ & $0.0033(9)$ & $-0.0014(8)$ \\
\hline C(19) & $8 f$ & $0.57302(9)$ & $0.0273(1)$ & $0.1071(1)$ & $0.0494(9)$ & $0.0393(9)$ & $0.0426(9)$ & $0.0064(8)$ & $0.0019(7)$ & $0.0013(7)$ \\
\hline$C(20)$ & $8 f$ & $0.6407(1)$ & $0.0319(2)$ & $0.0911(1)$ & $0.056(1)$ & $0.051(1)$ & $0.059(1)$ & $0.0147(9)$ & $0.0116(9)$ & $0.0043(9)$ \\
\hline$C(21)$ & $8 f$ & $0.6696(1)$ & $0.1136(2)$ & $0.0830(2)$ & $0.049(1)$ & $0.063(1)$ & $0.072(1)$ & $0.009(1)$ & $0.0203(9)$ & $0.010(1)$ \\
\hline$C(22)$ & $8 f$ & $0.6310(1)$ & $0.1916(1)$ & $0.0874(2)$ & $0.053(1)$ & $0.047(1)$ & $0.072(1)$ & $-0.0015(9)$ & $0.0175(9)$ & $0.0120(9)$ \\
\hline$C(23)$ & $8 f$ & $0.53859(9)$ & $0.1090(1)$ & $0.1133(1)$ & $0.0439(9)$ & $0.0336(8)$ & $0.0405(8)$ & $0.0010(7)$ & $0.0039(6)$ & $-0.0002(6)$ \\
\hline$C(24)$ & $8 f$ & $0.47006(8)$ & $0.1085(1)$ & $0.1320(1)$ & $0.0425(8)$ & $0.0343(9)$ & $0.0385(8)$ & $-0.0004(7)$ & $0.0011(6)$ & $-0.0025(6)$ \\
\hline
\end{tabular}

\section{References}

1. Wang, R. Z.; Xu, J. Q.; Yang, G. Y.; Bu, W. M.; Xing, Y. H.; Li, D. M.; Liu, S. Q.; Ye, L.; Fan, Y. G.: Structure of $\left[\mathrm{Cu}(\text { phen })_{2}\right]_{2}\left[\left\{\mathrm{Cu}(\mathrm{phen})_{2}\right\}-\right.$ $\mathrm{Mog}_{26}$ ] H2 O. Pol. J. Chem. 73 (1999) 1909-1916.

2. Healy, P. C.; Engelhandt, L. M.; Patrick, V. A.; White, A. H.: Lewis-base adducts of Group $1 B$ metal(I) compounds. Part 19. Crystal structures of bis(1,10-phenanthroline)copper(I) perchlorate and dibromocuprate(I). J. Chem. Soc., Dalton Trans. (1985) 2541-2545.

3. Sun, D. F.; Cao, R.; Hong, M. C.; Su, W. P.; Weng, J. B.: $\left(\mu_{2}-\right.$ Terephthalato)-bis[(bis(1,10-phenanthroline)-copper(I)] diperchlorate. Acta Crystallogr C56 (2000) e240-e241.

4. Dunal-Jurco, M.; Potocnak, I.; Kabesova, M.; Kettmann, U.; Miklos, D.: Mixed-valence $\mathrm{Cu}^{1}-\mathrm{Cu}^{11}$ complexes. Structure of cyanobis(1,10-phenanthroline)copper(II) bis(cyano)(1,10-phenanthroline)cuprate(I) pentahydrate. Acta Crystallogr. C49 (1993) 1479-1482.

5. Perkinson, J.; Brodie, S.; Yoon, K.; Mosny, K.; Carroll, P. J.; Morgan, T. V.; Burgmayer, S. J. N.: Preparations and properties of transition-metal pterin complexes. Models for the metal site in phenylalanine hydroxylase. Inorg. Chem. 30 (1991) 719-721.

6. Clarke, R.; Latham, K.; Rix, C.; White, J.: Two polymorphs of bis $(1,10-$ phenanthroline- $\left.\kappa^{2} N, N^{\prime}\right)$ copper(I) iodide. Acta Crystallogr. C59 (2003) m7-ms.

7. Pallenberg, A. J.; Koenig, S. K.; Barmhart, D. M.: Synthesis and Characterization of Some Copper(I) Phenanthroline Complexes. Inorg. Chem. 34 (1995) 2833-2840.
8. Klemens, F. K.; Palmer, C. E. A.; Rolland, S. M.; Fanwick, P. E.; McMillin, D. R.; Savage J.-P.: The crystal and molecular structures of the dpp ligand and $\left[\mathrm{Cu}(\mathrm{dpp})_{2}\right]\left[\mathrm{CuCl}_{2}\right] \cdot 1 / 2 \mathrm{CH}_{3} \mathrm{CN}$. New J. Chem. 14 (1990) 129-133.

9. Darensbourg, D. J.; Longridge, E. M.; Atnip, E. V.; Reibenspies, J. H.: A linear, monomeric copper(I) acetate derivative, bis[bis(phenanthroline) copper] bis(acetato)cuprate(1-) hydrogen bis(acetate). An effective catalyst for the decarboxylation of carboxylic acids. Inorg. Chem. 31 (1992) 3951-3955.

10. Yang, H. F.; Huang, C. C.; Zhang, H. H.; Liu, Y. X.; Lian, Z. X.; Xiao, G. C.: A new mixed-valence $\mathrm{Cu} / \mathrm{Cu}$ complex: chlorobis(1,10-phenanthroline)copper(II) dichlorocuprate(I). Acta Crystallogr. E60 (2004) m291-m293.

11. Yu, J. H.; Xu, J. Q.; Han, L.; Wang, T. G.; Shi, Z.; Jing, W. J.; Ding, H.; Xu, J. N.; Jia, H. B.; Hua, J.: Hydrothermal syntheses, supramolecular structures and the third-order non linear optical properties of three copper(I) halide amine complexes connected via secondary bonding interactions. Chin. J. Chem. 20 (2002) 851-857.

12. Sheldrick, G. M.: SHELXS-97. Program for the Solution of Crystal Structures. University of Göttingen, Germany 1997.

13. Sheldrick, G. M.: SHELXL-97. Program for the Refinement of Crystal Structures. University of Göttingen, Germany 1997. 